\title{
EXISTENCE THEOREMS FOR A FREE BOUNDARY PROBLEM IN COMBUSTION THEORY
}

\author{
BY \\ ROBERTO GIANNI AND PAOLA MANNUCCI \\ Università di Firenze, Firenze, Italy
}

0. Introduction. In this paper we analyze a one-dimensional parabolic partial differential equation which models a large number of physical phenomena. These are phenomena which, viewed on an appropriate time-scale, exhibit a switch-like behaviour. For this reason we have a source term in the equation that is discontinuous as a function of the dependent variable with a jump discontinuity.

This kind of problem was investigated by Norbury and Stuart in [1] where the equation was derived to model a combustion problem in a porous medium. In further papers, [4, 6, 7], Norbury and Stuart studied some mathematical aspects of such an equation (travelling waves, steady solutions, stability, and asymptotics). In [9], Friedman and Tzavaras proved the existence of a weak solution for the complete system of equations proposed in [1] to describe combustion problems.

The aim of this paper is to prove a global existence theorem of a classical solution having a "regular" free boundary, that is, a curve $x=s(t)$ which separates the domain in which we study the problem into two regions and through which the source term exhibits a jump.

The problem that we study, in the standard functional space, is:

$$
\begin{array}{ll}
u_{t}-\left(K(u) u_{x}\right)_{x}=f(u) \mathbb{H}(u-1) & \text { in } Q, \\
u(x, 0)=\phi(x) & \text { in }(0,1), \\
\mathscr{A} u(0, t)+\mathscr{B} u_{x}(0, t)=g_{1}(t) & \text { in }(0, T), \\
\mathscr{C} u(1, t)+\mathscr{D} u_{x}(1, t)=g_{2}(t) & \text { in }(0, T),
\end{array}
$$

where $Q=(0,1) \times(0, T) ;(\mathscr{A}, \mathscr{B}),(\mathscr{C}, \mathscr{D})$ are either $(0,1)$ or $(1,0)$.

In the problem studied by Norbury and Stuart, which will be our reference model, $u(x, t)$ is the temperature of a porous medium. $\mathbb{H}(*)$ is the Heaviside function, that we define to be zero when its argument is nonpositive, and one otherwise.

The interface is the level set $\mathfrak{S}=\{(x, t): u(x, t)=1\}$.

We look for a solution for which $\mathfrak{S}$ is a regular curve $x=s(t)$ such that $u_{x}(x, t)$ and $u_{t}(x, t)$ are continuous across $\{x=s(t)\}$; this means that the discontinuity, due to the presence of a discontinuous source term in the equation, appears only on

Received January 7, 1991.

1991 Mathematics Subject Classification. Primary 35K57, 35R35, 80A25.

Work partially supported by the Strategic Project "Mathematical Models for Industry" of the Italian C.N.R.

(C)1993 Brown University 
the second derivative of $u(x, t)$ with respect to $x$. A very large family of equations, which includes this problem, was studied by Fasano and Primicerio in [2], but this was done only in a sufficiently small interval of time $\left[0, t_{0}\right]$. Instead, in this paper, the result concerns two main issues: we find the existence of a regular solution $u(x, t)$ and we specify conditions under which we have a unique regular interface corresponding to the solution $u(x, t)$. This interface is global in time in the sense that it is regular in a time interval $[0, \bar{T}]$ in which it does not touch the lateral boundary $\{x=0\}$ and $\{x=1\}$. Actually, from the theorems of this paper, we can easily conclude that the interface $\mathfrak{S}$ is locally representable by a regular curve $x=s(t)$ in any inner compact domain $Q^{\prime} \subset Q$. As far as the regularity of the interface is concerned, we prove that $s(t)$ is Lipschitz continuous in $[0, \bar{T})$, continuous in $[0, \bar{T}]$ and Hölder continuous in $(0, \bar{T})$. (More generally, we prove that $\mathfrak{S}$ is locally representable by a curve $x=s(t)$ with $s(t) \in H^{1+\alpha}$ in any inner compact domain $Q^{\prime} \subset Q$.)

The paper is organized as follows. In the first section we investigate the global existence of a regular interface separating $Q$ into two regions $(u>1 ; u<1)$, where $u(x, t)$ is a solution of $(0.1)$, for a particular determination of the pairs $(\mathscr{A}, \mathscr{B})$ and $(\mathscr{C}, \mathscr{D})$. To get this result, we find some auxiliary estimates that are used to solve our problem. Moreover, a quite general local existence result is obtained.

In Sec. 2 we consider a certain number of problems that can be solved using the techniques of Sec. 1 and we give a more general local existence theorem.

Throughout the paper the notations are the same as in [3]. Moreover, with $\beta$ and $\gamma$ we denote any suitable Hölder exponents that we need not specify.

1. Global existence for a particular boundary condition. As a first step we consider the following problem as a tool to prove the theorems of the following section:

$$
\begin{gathered}
u_{t}-\left(K(u) u_{x}\right)_{x}=f(u) \mathbb{H}(u-1) \quad \text { in } Q, \\
u(x, 0)=\phi(x) \quad \text { in }(0,1), \\
u_{x}(0, t)=h(t) \quad \text { in }(0, T), \\
u_{x}(1, t)=k(t) \quad \text { in }(0, T) .
\end{gathered}
$$

We assume:

$$
\begin{aligned}
& K(u) \in C^{2}(\mathbb{R}) ; \quad K(u) \geq \widehat{K}>0, \\
& f(u) \in C^{1}(\mathbb{R}) ; \quad f(u)>0 \quad \text { for } u \geq 1, \\
& f(u) \text { grows as a linear function at infinity, } \\
& h(t), k(t) \in C^{1}([0, T]), \\
& \phi^{\prime}(x) \text { is Lipschitz continuous in }[0,1] \text { and } \\
& \quad \phi(x)>1 \text { if } x \in[0, b), \quad \phi(x)<1 \text { if } x \in(b, 1], \\
& \left|\phi^{\prime \prime}\right| \leq M \quad \text { in }[0,1] . \\
& \phi^{\prime}(0)=h(0), \quad \phi^{\prime}(1)=k(0) .
\end{aligned}
$$


THEOREM 1.1. Under the assumptions (1.5), there exists at least one function $u(x, t)$ such that

$$
\begin{aligned}
& u(x, t), u_{x}(x, t) \in H^{\beta, \beta / 2}(\bar{Q}) \text { for a suitable } \beta \\
& u(x, t) \in W_{q}^{2,1}(Q) \text { for all } q>2 \text { and satisfies (1.1)(a.e.), (1.2)-(1.4). }
\end{aligned}
$$

To prove this theorem let us consider a sequence of approximating problems:

$$
\begin{gathered}
u_{n t}-\left(K\left(u_{n}\right) u_{n x}\right)_{x}=f\left(u_{n}\right) \mathbb{H}_{n}\left(u_{n}-1\right) \quad \text { in } Q, \\
u_{n}(x, 0)=\phi_{n}(x) \quad \text { in }(0,1), \\
u_{n x}(0, t)=h_{n}(t) \quad \text { in }(0, T), \\
u_{n x}(1, t)=k_{n}(t) \quad \text { in }(0, T) .
\end{gathered}
$$

Here $h_{n}(\eta), k_{n}(\eta), \phi_{n}(\eta) \in C^{\infty}(\mathbb{R})$ and they converge to their respective limits $h, k, \phi$ in the $C^{1}$-norm; moreover, we can arrange that $\phi_{n}(x)-1$ changes sign only at $b$. For $\mathbb{H}_{n}(\eta)$ we have

$$
\begin{aligned}
& \mathbb{H}_{n}(\eta) \in C^{\infty}(\mathbb{R}), \\
& \lim _{n}\left\|\mathbb{H}_{n}-\mathbb{H}\right\|_{C^{2}}=0 \quad \text { in } \mathbb{R} \backslash(-\varepsilon, \varepsilon) \forall \varepsilon>0, \\
& \lim _{n}\left\|\mathbb{H}_{n}-\mathbb{H}\right\|_{2, \mathbb{R}}=0, \\
& \mathbb{H}_{n}(\eta) \text { is an increasing function of its argument. }
\end{aligned}
$$

Moreover, because of assumptions (1.5) it is possible to choose $\phi_{n}(x)$ such that

$$
\left|\phi_{n}^{\prime \prime}\right| \leq M \text {. }
$$

Here and in the following, $M$ will denote any constant independent of $n$. We assume the system (1.7)-(1.10) satisfies the first-order compatibility conditions. The existence of a classical solution of problem (1.7)-(1.10) follows from [3, Theorem (7.4), p. 491] once we have applied the Kirchoff transformation:

$$
v_{n}(x, t)=\int_{0}^{u_{n}(x, t)} K(\eta) d \eta .
$$

Proposition 1.2. The following estimates hold:

$$
\begin{gathered}
\max _{Q}\left|u_{n}\right| \leq M, \\
\left|u_{n}\right|_{Q}^{(1+\beta)} \leq M, \\
\left\|u_{n x x}, u_{n t}\right\|_{q, Q} \leq M .
\end{gathered}
$$

Proof. The estimate (1.12) follows from [3, Theorem (7.3), p. 487] (using the Kirchoff transformation (1.11)). Estimates (1.13) and (1.14) follow from [3, Theorem (1.1), p. 419; Theorem (3.1), p. 437]. Although these theorems give an estimate in an inner domain of the form $(\varepsilon, 1-\varepsilon) \times(0, T)$, we can overcome this difficulty in the following way. We reflect the domain $Q$ with respect to $x=0$ and $x=1$, 
respectively. Then we apply the previous theorems to the two parabolic problems satisfied by the functions

$$
\bar{v}_{n}(x, t)=v_{n}(x, t) \quad \text { if } x \in[0,1], \quad \bar{v}_{n}(x, t)=v(-x, t) \quad \text { if } x \in[-1,0),
$$

and

$$
\bar{w}_{n}(x, t)=w_{n}(x, t) \quad \text { if } x \in[0,1], \quad \bar{w}(x, t)=w_{n}(2-x, t) \quad \text { if } x \in(1,2],
$$

where

$$
v_{n}(x, t)=u_{n}(x, t)-x h_{n}(t), \quad w_{n}(x, t)=u_{n}(x, t)-(1-x) k_{n}(t) .
$$

Thus we get the estimates up to the boundary.

Proof of Theorem 1.1. Let $u_{n}(x, t)$ be the solution of problem (1.7)-(1.10). The estimates (1.12)-(1.14) are independent of $n$, and we can extract a subsequence from $\left\{u_{n}\right\}$, that we denote again by $\left\{u_{n}\right\}$, which converges in the following sense:

$$
\begin{gathered}
u_{n} \stackrel{C^{0}}{\longrightarrow} u \in H^{\beta, \beta / 2} \quad \text { in } \bar{Q}, \quad u_{n x} \stackrel{C^{0}}{\longrightarrow} u_{x} \in H^{\beta, \beta / 2} \quad \text { in } \bar{Q}, \\
u_{n x x}, u_{n t} \stackrel{\mathrm{w}-L^{2}}{\longrightarrow} u_{x x}, u_{t} \text { in } Q,
\end{gathered}
$$

(here and in the following w- $L^{2}$ denotes weak convergence in $L_{2}$ ).

Applying to (1.1)-(1.4) Theorem (9.1) on p. 341 of [3], we get that $u(x, t) \in$ $W_{q}^{2,1}(Q) \forall q$. Rigorously speaking, it is not immediately clear that

$$
\mathbb{H}_{n}\left(u_{n}-1\right) \stackrel{\mathrm{w}-L^{2}}{\longrightarrow} \mathbb{H}(u-1) \text {. }
$$

However, we can easily obtain that

$$
\mathbb{H}_{n}\left(u_{n}-1\right) \stackrel{\mathrm{w}-L^{2}}{\longrightarrow} \Phi(x, t), \quad \Phi(x, t) \in L_{\infty}(Q)
$$

with $\Phi(x, t)=1$ if $u(x, t)>1 ; \Phi(x, t)=0$ if $u(x, t)<1$, and $0 \leq \Phi(x, t) \leq 1$ in $\mathfrak{S}:=\{(x, t) \in Q: u(x, t)=1\}$. However, it can be proved that either meas $\mathfrak{S}=0$ or $\Phi(x, t)=0$ a.e. in $\mathfrak{S}$. In fact, using Lemma A.4 on p. 53 of [8], since $u_{x}(x, t)$ and $u_{t}(x, t)$ are in $L_{2}(Q)$, we get $u_{x}(x, t) \equiv u_{t}(x, t) \equiv 0$ a.e. in $\mathfrak{S}$. At this point, still applying Lemma A.4 on p. 53 of [8], to the function $u_{x}(x, \bar{t})$ (remember that because of Fubini's Lemma, $u_{x x}(x, \bar{t}) \in L_{2}((0,1))$ for almost every $\left.\bar{t}\right)$, we get $u_{x x}(x, \bar{t}) \equiv 0$ in $\mathfrak{S} \cap\{t=\bar{t}\}$. Hence $u_{x x}(x, t) \equiv 0$ a.e. in $\mathfrak{S}$. Since $u_{t}-u_{x x}=\Phi$ a.e. in $Q$, we have that $\Phi(x, t) \equiv 0$ a.e. in $\mathfrak{S}$; then $\Phi \equiv \mathbb{H}(u-1)$ and this completes the proof.

This implies the existence of a solution $u(x, t)$ for the system (1.1)-(1.4).

THEOREM 1.3. Under the assumptions (1.5), if

$$
\begin{gathered}
h(t) \leq \delta<0, \quad k(t) \leq \delta<0, \quad \phi^{\prime}(x) \leq \delta<0, \\
K^{\prime}(u) \geq 0, \quad f^{\prime}(u) \geq 0 ;
\end{gathered}
$$

then

$$
\begin{gathered}
u_{x}(x, t) \leq \delta<0 \quad \text { in } \bar{Q} \\
u_{t}(x, t) \in H^{\beta, \beta / 2}(Q), \\
\left|u_{t}(x, t)\right| \leq M \quad \text { in } \bar{Q}
\end{gathered}
$$


Proof. We consider again the approximating problems (1.7)-(1.10), selecting data so that they satisfy inequalities of the same kind as (1.15) uniformly in $n$. Let us set $w_{n}(x, t)=v_{n x}(x, t)$ where $v_{n}(x, t)$ is defined by (1.11). The function $w_{n}(x, t)$ satisfies the following system of equations:

$$
\begin{aligned}
& w_{n t}-\widehat{K}\left(v_{n}\right) w_{n x x}-\widehat{K}^{\prime}\left(v_{n}\right) w_{n} w_{n x}=\left(\hat{f}\left(v_{n}\right) \widehat{\mathbb{H}}_{n}\left(v_{n}\right) \widehat{K}\left(v_{n}\right)\right)^{\prime} w_{n} \quad \text { in } Q, \\
& w_{n}(x, 0)=\widehat{K}\left(v_{n}(x, 0)\right) \phi_{n}^{\prime}(x) \leq \delta<0 \text { in }(0,1), \\
& w_{n}(0, t)=\widehat{K}\left(v_{n}(0, t)\right) h_{n}(t) \leq \delta<0 \text { in }(0, T), \\
& w_{n}(1, t)=\widehat{K}\left(v_{n}(1, t)\right) k_{n}(t) \leq \delta<0 \text { in }(0, T),
\end{aligned}
$$

where the prime in the right side of the parabolic equation denotes differentation with respect to $v_{n}$. Here $\widehat{K}\left(v_{n}\right)=K\left(u_{n}\left(v_{n}\right)\right)$ and $\hat{f}\left(v_{n}\right)=f\left(u_{n}\left(v_{n}\right)\right.$ ) (where $u_{n}\left(v_{n}\right)$ is the inverse function of (1.11)).

It is easy to verify that, because of the assumptions, $\widehat{K}^{\prime}(\eta)$ and $\hat{f}^{\prime}(\eta)$ are nonnegative. Then, from the maximum principle, we see that $w_{n}(x, t) \leq 0$ on $\bar{Q}$. Regarding $\left(\hat{f}\left(v_{n}\right) \widehat{\mathbb{H}}_{n}\left(v_{n}\right) \widehat{K}\left(v_{n}\right)\right)^{\prime} w_{n}$ as a nonpositive source term and applying the strong maximum principle, we get:

$$
u_{n x}(x, t) \leq M<0 \text { on } Q,
$$

with $M$ a suitable negative constant independent of $n$.

Let us now prove the Hölder continuity of $u_{n t}$. The first step is to prove that

$$
\sup _{Q}\left|u_{n t}(x, t)\right| \leq M \text {. }
$$

Let $z_{n}(x, t)=u_{n t}(x, t) . z_{n}(x, t)$ satisfies the following equations:

$$
\begin{gathered}
z_{n t}-\left(K\left(u_{n}\right) z_{n x}-K^{\prime}\left(u_{n}\right) z_{n} u_{n x}\right)_{x}-\left(f^{\prime} \mathbb{H}_{n}+f \mathbb{H}_{n}^{\prime}\right) z_{n}=0, \\
z_{n}(x, 0)=\left(K\left(\phi_{n}(x)\right) \phi_{n}^{\prime}(x)\right)^{\prime}+\mathbb{H}_{n}\left(\phi_{n}-1\right) .
\end{gathered}
$$

We prove now that the assumptions of Theorem (8.1) on p. 192 of [3] are satisfied. This will imply the estimate (1.20). Because of (1.12)-(1.14), we need only to prove that

$$
\left\|f^{\prime}\left(u_{n}\right) \mathbb{H}_{n}\left(u_{n}-1\right)+f\left(u_{n}\right) \mathbb{H}_{n}^{\prime}\left(u_{n}-1\right)\right\|_{q, r, Q} \leq M
$$

with $q=1$ and suitable $r \quad(r \neq \infty)$. We have

$$
\begin{aligned}
\| f^{\prime} \mathbb{H}_{n}+f \mathbb{H}_{n}^{\prime} !_{1, r, Q} & =\left\{\int_{0}^{T}\left(\int_{0}^{1}\left|f^{\prime} \mathbb{H}_{n}+f \mathbb{H}_{n}^{\prime}\right| d x\right)^{r} d t\right\}^{1 / r} \\
& \leq\left\{\int_{0}^{T}\left(\int_{0}^{1}\left|f^{\prime} \mathbb{H}_{n}\right| d x+\int_{0}^{1}\left|f \mathbb{H}_{n}^{\prime}\right| d x\right)^{r} d t\right\}^{1 / r} .
\end{aligned}
$$

Now we multiply and divide $\mathbb{H}_{n}^{\prime}$ by $u_{n x}$ and use (1.12), (1.19) to obtain

$$
\begin{aligned}
\left\|f^{\prime} \mathbb{H}_{n}+f \mathbb{H}_{n}^{\prime}\right\|_{1, r, Q} & \leq\left\{\int_{0}^{T}\left(C_{1}+C \int_{0}^{1} \frac{d \mathbb{H}_{n}\left(u_{n}-1\right)}{d x} d x\right)^{r} d t\right\}^{1 / r} \\
& \leq\left\{\int_{0}^{T}\left(C_{1}+C\right)^{r} d t\right\}^{1 / r} \leq \widehat{C} T^{1 / r}
\end{aligned}
$$


This allows us to get an estimate for $\left|z_{n}\right|$ in any inner domain of $Q$.

At this point, by means of the same arguments (reflection with respect to $\{x=0\}$ and $\{x=1\})$ used to prove the estimates (1.13)-(1.14) and again using Theorem (8.1) on p. 192 of [3], we obtain (1.20) (remember that $z_{n}$ is bounded independently of $n$ on the parabolic boundary of $Q)$. Using estimate (1.20) and Theorem (10.1) on p. 204 of [3] we get that, $\forall \varepsilon$ :

$$
\left|u_{n t}(x, t)\right|^{(\beta)} \leq M\left(Q^{\prime}\right) \text { in } Q^{\prime} \text {, uniformly on } n,
$$

where $Q^{\prime}$ is of the form $Q^{\prime}=[\varepsilon, 1-\varepsilon] \times[\varepsilon, T]$. Eventually, using the same argument used to prove Theorem 1.1 (convergence of a subsequence $\left\{u_{n k}\right\} \subset\left\{u_{n}\right\}$ to a solution $u$ of our problem), we get (1.16)-(1.18).

THEOREM 1.4. Under the assumptions (1.5) and (1.15) there exists a solution to problem (1.1)-(1.4) such that

$u(x, t), u_{x}(x, t) \in H^{\beta, \beta / 2}(\bar{Q}), u_{t}(x, t) \in H^{\beta, \beta / 2}(Q)$, for a suitable $\beta \in(0,1)$,

$s(t) \in H^{1+\gamma}((0, T])$, for a suitable $\gamma \in(0,1), s(t)$ is Lipschitz continuous in $[0, T]$, where $u(s(t), t)=1$ with $t \in[0, T]$.

(Here and in the following sections when we write that $s(t) \in H^{1+\gamma}((0, T])$, $\forall T>0$, we mean that this is true whilst the problem is really a two-phase problem; that is, if $\forall \bar{t} \in[0, T]$, none of the two sets $\{x \in[0,1] ; u(x, \bar{t}) \geq 1\},\{x \in$ $[0,1] ; u(x, \bar{t}) \leq 1\}$ is void.)

More generally, the theorem implies that the interface $\mathfrak{S}$ is locally representable by a curve $x=s(t) \in H^{1+\gamma}$ in any inner compact domain $Q^{\prime} \subset Q$. This result holds true for all the theorems below and therefore it will not be stated again.

Proof. Using Theorem 1.1 and estimates (1.16)-(1.18), and by means of the inverse function theorem, we get that the level set $\{(x, t) \in Q: u(x, t)=1\}$ can be represented by a function of the form $x=s(t)$ for $t \in[0, T]$ with $s(t) \in H^{1+\gamma}((0, T])$ for a suitable $\gamma$ and $s(t)$ Lipschitz continuous in $[0, T]$. We stress the fact that, for the existence of this curve the condition $u_{x}(x, t) \leq \delta<0$ on $Q$ is crucial. At this point the other estimates of Theorem 1.4 follow from Theorems 1.1 and 1.3.

Clearly only one curve starts from $\{t=0\}$ because, from the assumptions (1.5) and (1.15), there is only one $b$ such that $\phi(b)=1$. Moreover, if a $\bar{t}$ exists such that $s(\bar{t})=0$ or $s(\bar{t})=1$, then the curve $s(t)$ can start again only from the axis $\{x=0\}$ or $\{x=1\}$, respectively. We can derive this from Hopf's theorem. Using again Hopf's theorem we get that no other level curve $u=1$ can start from the axes $x=0$ and $x=1$ while the curve from $(b, 0)$ is still present. This implies that until there is a region $\{u \geq 1\}$ and a region $\{u \leq 1\}$, there exists a curve $s(t)$ that separates the two regions and the curve is unique in the sense that for any $t=t_{0}$ a unique point $x_{0}$ exists such that $u>1$ in $0<x<x_{0}, u<1$ in $x_{0}<x<1$, and $x_{0}=s\left(t_{0}\right)$.

REMARK 1.1. It is easy to observe that we can drop all the assumptions (1.15) if we are interested in a local existence theorem. For the sake of simplicity we assume that there exists a unique $b$ such that $\phi(b)=1$ and $\phi^{\prime}(b)<0$ (the last condition is quite natural because we need $\phi^{\prime}(b) \neq 0$ if we want to have a classical solution up to $\{t=0\})$. 
First we can prove the existence of a weak solution as in Theorem 1.1. Then using the Hölder continuity of $u_{x}(x, t)$, we get $u_{x}(x, t) \leq \delta<0$ on $(b-\varepsilon, b+\varepsilon) \times(0, \tau)$ for suitable $\delta, \varepsilon$, and $\tau$. At this point we can proceed as in Theorem 1.1, obtaining the existence of a regular interface in $(b-\varepsilon, b+\varepsilon) \times(0, \tau)$, eventually proving the local existence theorem. We observe that this local existence theorem is rather general, because it allows us to make only standard assumptions on the regularity on the boundary data and on the coefficients of the equation, the stronger assumptions being made only for $\phi(x)$ in the vicinity of $x=b$.

2. Other cases of global existence. Let us now deal in more detail with the problem with no flux boundary condition at $x=0$, i.e.,

$$
\begin{array}{cl}
u_{t}-\left(K(u) u_{x}\right)_{x}=f(u) \mathbb{H}(u-1) & \text { in } Q, \\
u(x, 0)=\phi(x) & \text { in }(0,1), \\
u_{x}(0, t)=0 & \text { in }(0, T), \\
u(1, t)=h(t) & \text { in }(0, T),
\end{array}
$$

with

$$
\begin{gathered}
\phi^{\prime}(b) \neq 0 ; \quad \phi^{\prime}(x) \leq 0 \quad \forall x \in(0,1), \\
h(t)<1, \quad h^{\prime}(t) \leq 0, \\
K^{\prime}(\eta) \geq 0, \quad f^{\prime}(\eta) \geq 0 .
\end{gathered}
$$

Let the assumptions on the regularity on $\phi(x), K(\eta)$, and $f(\eta)$ be the same as in Theorem (1.1). Here, as before, $T$ is the largest interval in which the problem is really a two-phase problem.

THEOREM 2.1. Under the assumptions (1.5) and (2.5) there exists a solution of (2.1)(2.4) with the same properties listed in Theorem 1.4 such that $s(t) \in H^{1+\gamma}((0, T))$, $s(t)$ is Lipschitz continuous in a neighbourhood of $x=b, t=0$, and $s(t)$ is continuous in $[0, T]$.

As in Sec. 1 we write down an approximating system of the form

$$
\begin{aligned}
u_{n t}-\left(K\left(u_{n}\right) u_{n x}\right)_{x} & =f\left(u_{n}\right) \mathbb{H}_{n}\left(u_{n}-1\right) \quad \text { in } Q, \\
u_{n}(x, 0) & =\phi_{n}(x) \quad \text { in }(0,1), \\
u_{n x}(0, t) & =l_{n}(t) \quad \text { in }(0, T), \\
u_{n}(1, t) & =h_{n}(t) \quad \text { in }(0, T),
\end{aligned}
$$

with $\phi_{n}^{\prime}(x) \leq 0, \phi_{n}(b)=1, \phi_{n}^{\prime}(b)>\delta>0, h_{n}^{\prime}(t) \leq 0$, and $l_{n}(t) \leq 0$, converging to zero uniformly. Moreover, the assumptions on the approximating system in Sec. 1 are still satisfied. To prove Theorem 2.1 we will use Propositions 2.2, 2.3, and 2.4.

Proposition 2.2. Under the assumptions (1.5), the problem (2.1)-(2.4) admits at least one weak solution $u(x, t)$ such that $u(x, t), u_{x}(x, t) \in H^{\beta, \beta / 2}(\bar{Q})$ for a suitable $\beta ; u(x, t) \in W_{q}^{2,1}(Q)$ for all $q>2$. 
Proof. We consider the system of equations satisfied by functions $\bar{v}_{n}(x, t)$ defined in $[-1,1] \times[0, T]$, which is equal to $v_{n}(x, t)$ in $[0,1]$ and to $v_{n}(-x, t)$ in $[-1,0]$, where $v_{n}(x, t)=u_{n}(x, t)-x l_{n}(t)$. Then we apply Theorem $(6.1)$ on $\mathrm{p} .457$ of [3] which gives us the existence of a regular solution of such a system and hence the existence of a regular solution of system (2.6)-(2.9). Moreover, from Theorem (2.2) on p. 428 of [3] we have $\max \left|u_{n x}\right| \leq M$.

From Lemma (3.1) on p. 535 and Theorem (4.1) on p. 443 of [3] we obtain

$$
\left|u_{n}\right|_{Q}^{(1+\beta)} \leq M, \quad\left\|u_{n x x}, u_{n t}\right\|_{q, Q} \leq M
$$

As in the proof of Theorem 1.1 we can now extract a subsequence $\left\{u_{k}\right\}$ that converges to a function $u(x, t)$ which is a solution of $(2.1)-(2.4)$ and which satisfies the properties we need.

Proposition 2.3. Under the assumptions (2.5) and (1.5), the solution $u(x, t)$ of problem (2.1)-(2.4) is such that

$$
\begin{gathered}
u_{x}(x, t) \leq \delta(\varepsilon)<0 \quad \text { in }(\varepsilon, 1) \times(\varepsilon, T) \forall \varepsilon>0, \\
\left|u_{t}\right|^{(\beta)} \leq M(\varepsilon) \quad \text { in }(\varepsilon, 1) \times(\varepsilon, T) \forall \varepsilon>0,
\end{gathered}
$$

with $\delta(\varepsilon)$ and $M(\varepsilon)$ independent of $n$.

Proof. Let us observe that $u_{n x}(1, t) \leq \delta_{1}<0 \quad \forall t \in(0, T)$, with $\delta_{1}$ independent of $n$. In fact, because of the assumptions on the boundary data, in $(0,1) \times(0, \bar{t})$, $u(x, t)$ takes its minimum at $(1, \bar{t})$, then by Hopf's theorem we get the result. Using the strong maximum principle, as in Theorem 1.3, we get

$$
u_{n x}(x, t) \leq \delta(\varepsilon)<0 \text { in }[\varepsilon, 1] \times[\varepsilon, T] \forall \varepsilon>0,
$$

uniformly on $n$ and hence (2.10) is obtained. At this point, proceeding as in Sec. 1 and using (2.12) we get

$$
\left|u_{n t}\right|^{(\beta)} \leq M(\varepsilon) \quad \text { in }(\varepsilon, 1) \times(\varepsilon, T) \forall \varepsilon>0,
$$

with $M(\varepsilon)$ independent of $n$. Now this implies that we can choose the subsequence $\left\{u_{k}\right\}$ of the proof of Theorem 2.2 such that

$$
u_{k t} \stackrel{{ }^{0}}{\longrightarrow} u_{t} \in H^{\beta, \beta / 2}([\varepsilon, 1] \times[\varepsilon, T]) \quad \forall \varepsilon .
$$

(This can be done using diagonal sequence.)

Proposition 2.4. Under the assumptions (1.5) and (2.5) there exist two positive constants $\hat{\varepsilon}$ and $\tau$ such that in the rectangle $[b-\hat{\varepsilon} / 2, b+\hat{\varepsilon} / 2] \times[0, \tau], u_{t}(x, t)$ is bounded and $u_{x}(x, t) \leq \delta_{2}<0$.

Proof. Since $\phi_{n} \stackrel{c^{\prime}}{\longrightarrow} \phi$, for some $\hat{\varepsilon}>0$ we have $\phi_{n}^{\prime}(x) \leq \delta_{3}<0$ in $[b-\hat{\varepsilon}, b+\hat{\varepsilon}]$. Using the uniform Hölder continuity of $u_{n x}$, we get that an analogous result is true in $[b-\hat{\varepsilon}, b+\hat{\varepsilon}] \times[0, \tau]$ for a suitable $\tau$, so, following the path of the proof in Sec. 1 , we get the uniform boundedness of $u_{n t}$ in $[b-\hat{\varepsilon} / 2, b+\hat{\varepsilon} / 2] \times[0, \tau]$. This implies that $\left|u_{t}\right| \leq M$ in $[b-\hat{\varepsilon} / 2, b+\hat{\varepsilon} / 2] \times[0, \tau]$.

Let us now return to Theorem 2.1. 
Proof of Theorem 2.1. Using Propositions 2.2, 2.3, 2.4, we get that the solution of the approximating problem converges to a function $u(x, t)$ that satisfies the properties we are looking for. Moreover, as done in Sec. 1 (Theorem 1.4), we get that a regular interface $x=s(t)$ for our solution exists such that $\dot{s}(t) \in H^{\gamma}((0, T))$ for a suitable $\gamma \in(0,1)$ and $s(t)$ is Lipschitz continuous in a neighbourhood of $x=b$, $t=0$. Moreover, $s(t)$ is continuous in $[0, T]$. In fact, if $\lim _{\sup _{t \rightarrow T}} s(t)=L$ with $L>0$, we have, because of the continuity, that $u(L, T)=1$ and this contradicts $u_{x}(x, t)<0$ in $(0,1) \times(0, T]$.

From the proofs of Theorems (1.4) and (2.1) we see that the crucial point in our global existence theorems for the interface is an estimate of the sign of $u_{x}(x, t)$ on the lateral boundary $x=0, x=1$.

For these reasons, still using an approximating system, it is not difficult to prove that analogous theorems are true for the problems

$$
\begin{gathered}
u_{t}-\left(K(u) u_{x}\right)_{x}=f(u) \mathbb{H}(u-1) \quad \text { in } Q, \\
u(x, 0)=\phi(x) \quad \text { in }(0,1),
\end{gathered}
$$

with the boundary conditions

$$
u(0, t)=g_{1}(t) \quad \text { in }(0, T), \quad u(1, t)=g_{2}(t) \quad \text { in }(0, T),
$$

with

$$
g_{1}^{\prime}(t) \geq \max |f|, \quad g_{2}^{\prime}(t) \leq 0 .
$$

(Here and below we assume, for the sake of simplicity, that $|f(\eta)|$ is bounded.)

$$
\begin{aligned}
& \begin{array}{l}
u_{x}(0, t)=g_{1}(t) \leq 0 \quad \text { in }(0, T), \quad \text { with } g_{2}^{\prime}(t) \leq 0 . \\
u(1, t)=g_{2}(t) \text { in }(0, T)
\end{array} \\
& u(0, t)=g_{1}(t) \text { in }(0, T) \text {, } \\
& u_{x}(1, t)=g_{2}(t) \leq 0 \quad \text { in }(0, T) \\
& \text { with } g_{1}^{\prime}(t) \geq \max |f| \text {. } \\
& u_{x}(0, t)=g_{1}(t) \leq 0 \quad \text { in }(0, T) \text {, } \\
& u_{x}(1, t)=g_{2}(t) \leq 0 \quad \text { in }(0, T) .
\end{aligned}
$$

The assumptions on the coefficients of the equation, on the boundary data, and on $\phi(x)$ are the same as the previous theorems (see for example (1.5), (1.15), (2.5)). At this point, by means of the maximum principle and Hopf's theorem, it is easy to prove that in all the previous cases $u_{x}(0, t) \leq 0, u_{x}(1, t) \leq 0$, and then we can proceed as in Theorem 2.1.

We can summarize the results above in the following statement.

THEOREM 2.5. Consider the following equations:

$$
\begin{aligned}
u_{t}-\left(K(u) u_{x}\right)_{x}=f(u) \mathbb{H}(u-1) & \text { in } Q, \quad u(x, 0)=\phi(x) \quad \text { in }(0,1), \\
+ & \text { boundary conditions on } x=0, \quad x=1,
\end{aligned}
$$

under the assumptions: $K(\eta) \in C^{2}(\mathbb{R}), f(\eta) \in C^{1}(\mathbb{R}), K^{\prime}(\eta) \geq 0, f^{\prime}(\eta) \geq 0 ; \phi^{\prime}(x)$ is Lipschitz continuous in $[0,1] ; \phi^{\prime}(x) \leq 0 ; \phi(b)=1 ; \quad \phi^{\prime}(b)<0 ;$ and $\left|\phi^{\prime \prime}\right| \leq M$ in $[0,1]$. Assume that an approximating system $\left(\mathrm{P}_{n}\right)$ of $(\mathrm{P})$ as in Theorem 1.1 exists such that $u_{n}^{\prime}(x, 0)=\phi_{n}^{\prime}(x) \leq 0, \phi_{n}^{\prime}(b)<\delta<0, \phi_{n}(b)=1 \quad \forall n$, and such 
that a weak solution $u_{n}$ of $\left(\mathrm{P}_{n}\right)$ exists for which $\left\|u_{n}\right\|_{2, Q} \leq M,\left|u_{n}\right|_{Q}^{(\beta)} \leq M$, $\left|u_{n x}\right|_{Q}^{(\beta)} \leq M$ and moreover $u_{n x}(0, t) \leq 0, u_{n x}(1, t) \leq 0$; then a solution $(u, s)$ of problem (P) exists with the same properties as in Theorem 2.1.

The proof of this theorem follows the same pattern as the one of Theorem 2.1 with some minor technical differences.

By means of Theorem 2.5 we can deal with a slightly more general case than (A), (B), (C), (D). For example, we can consider the boundary conditions $u_{x}(0, t)=$ $K(u, t) ; u_{x}(1, t)=\widehat{K}(u, t)$ with $K \leq 0 ; \widehat{K} \leq 0$, sufficiently "regular".

REMARK 2.1. Theorems on the existence of a solution $u(x, t)$ having a "regular" interface, for all the systems of equations considered so far, are valid also if $\phi^{\prime}(b)=0$ ( $b$ is still the unique point in which $\phi(b)=1$ ). The assumptions on the boundary conditions and on the coefficients are the same as the previous cases. In fact once we have written down an approximate system as in Theorem 1.1 whose solution $u_{n}(x, t)$ satisfies estimates of the form

$$
\left\|u_{n}\right\|_{2, Q} \leq M, \quad\left|u_{n}\right|_{Q}^{(\beta)} \leq M, \quad\left|u_{n x}\right|_{Q}^{(\beta)} \leq M,
$$

we can proceed as in Theorem 2.1, obtaining

$$
u_{n x}(x, t) \leq \delta(\varepsilon)<0 \quad \text { in } Q^{\prime} \quad \forall \varepsilon>0, \quad\left|u_{n t}\right|_{Q^{\prime}}^{(\beta)} \leq M(\varepsilon) \quad \forall \varepsilon>0,
$$

where

$$
Q^{\prime}=[\varepsilon, 1-\varepsilon] \times[\varepsilon, T],
$$

with $\delta(\varepsilon)$ and $M(\varepsilon)$ independent of $n$. This implies the existence of a solution $u(x, t)$ for the original system such that

$$
u_{x}(x, t) \leq \delta(\varepsilon)<0 \quad \text { in } Q^{\prime} \quad \forall \varepsilon>0, \quad\left|u_{t}\right|_{Q^{\prime}}^{(\beta)} \leq M(\varepsilon),
$$

and then the set $\mathfrak{S}=\{(x, t) \in Q: u(x, t)=1\}$ is representable as a regular curve $s(t) \in H^{1+\gamma}((0, T])$, for a suitable $\gamma \in(0,1)$. As $t$ goes to zero we have that $s(t)$ goes to $b$. In fact if a sequence $\left\{t_{n}\right\}$ exists such that $s\left(t_{n}\right)$ goes to $x_{0} \neq b$ we get, because of the continuity of $u(x, t), u\left(x_{0}, 0\right)=1$ and so $\phi(x)=1 \quad \forall x \in\left(x_{0}, b\right)$ or $\left(b, x_{0}\right)$ and this is against our assumptions. So, by the technique of Theorem 1.1 and Theorem 2.1, we have proved also in this case the existence of an interface $s(t) \in H^{1+\gamma}((0, T]) \cap C^{0}([0, T]) \quad(\forall T$ for which we have a two-phase problem in $[0,1] \times[0, T])$.

REMARK 2.2. As in Remark 1.1, we can drop all the assumptions on the sign of $K^{\prime}(\eta), f^{\prime}(\eta), \phi^{\prime}(x)$ and on the boundary conditions (only keeping $\phi^{\prime}(b) \neq 0$ ) if we want just local existence. The same can be done when $\phi^{\prime}(b)=0$ and $\phi^{\prime}(x)<0$ in $[b-\varepsilon, b) \cup(b, b+\varepsilon]$ but in this case we still need the assumptions $K^{\prime}(\eta) \geq 0$, $f^{\prime}(\eta) \geq 0$.

In conclusion, we see that if we have a problem that can be treated as a free boundary problem because of the discontinuity on the source term, it can be useful to solve it simply by looking for a weak solution and regularizing it up to a point such that we can apply the inverse function theorem. For the problems solved in this paper, this technique allows us to get global results on the existence of the free boundary. Moreover, this technique gives a very simple way to get a local existence 
theorem for a solution having a regular free boundary under very general assumptions on the coefficients and on the boundary data.

\section{REFERENCES}

[1] J. Norbury and A. M. Stuart, A model for porous medium combustion, Quart. J. Mech. Appl. Math. 42, 159-178 (1987)

[2] A. Fasano and M. Primicerio, Classical solutions of general two-phase parabolic free boundary problems in one dimension, Free Boundary Problems: Theory and Applications, vol. II (A. Fasano and M. Primicerio, eds.), Pitman Advanced Publishing Program, Boston, London, and Melbourne, 1981

[3] O. A. Ladyzhenskaya, V. A. Solonnikov, and N. N. Ural' ceva, Linear and quasilinear equations of parabolic type, Izdat. "Nauka", Moscow, 1967, Translations of Mathematical Monographs, Vol. 23, Amer. Math. Soc., Providence, RI, 1968

[4] J. Norbury and A. M. Stuart, Parabolic free boundary problems arising in porous medium combustion, IMA J. Appl. Math. 39, 241-257 (1987)

[5] A. Friedman, Partial differential equations of parabolic type, Prentice-Hall, Englewood Cliffs, N.J., 1964

[6] J. Norbury and A. M. Stuart, Travelling combustion waves in a porous medium. Part 1, Existence, SIAM J. Appl. Math. 48, 155-169 (1988)

[7] J. Norbury and A. M. Stuart, Travelling combustion waves in a porous medium. Part 2, Stability, SIAM J. Appl. Math. 48, 374-392 (1988)

[8] D. Kinderlehrer and G. Stampacchia, An introduction to variational inequalities and their applications, Academic Press, New York, London, and Toronto, 1980

[9] A. Friedman and A. Tzavaras, Combustion in a porous medium, SIAM J. Math. Anal. 19, 509-519 (1988) 